\title{
Common laboratory tests as indicators of COVID-19 severity on admission at high altitude: a single-center retrospective study in Quito (ECUADOR)
}

https://doi.org/10.1515/cclm-2021-0156

Received January 30, 2021; accepted February 23, 2021; published online March 5, 2021

Keywords: biomarkers; clinical laboratory techniques; COVID-19; epidemiology.

To the Editor,

The current outbreak of SARS-Cov-2, a virus responsible for the coronavirus disease (namely COVID-19) in Wuhan (CHINA), has infected 107.1 million and caused over 2.34 million deaths worldwide (https://www.worldometers. info/coronavirus/). The main symptoms after infection are fever, dry cough, and fatigue, although disease severity can increase thereafter showing strong inter-individual differences. At worst, severe cases (4.7-6.1\%) quickly progress to an acute respiratory distress syndrome (ARDS), septic shock, difficult-to-correct metabolic acidosis, coagulation dysfunction, and multiple organ failure. The fatality rate indeed reaches a $61.5 \%$ of the critically ill patients. In the fight against the coronavirus pandemic, prediction of disease severity is an urgent clinical need. COVID-19 prognosis

\footnotetext{
*Corresponding author: Prof. Martha Fors, PhD, Escuela de Medicina, Universidad de Las Américas-UDLA, Quito, Ecuador, E-mail: martha.fors@udlanet.ec. https://orcid.org/0000-00020844-199X

Santiago J. Ballaz, Escuela Ciencias Biológicas e Ingeniería, Universidad Yachay Tech, Urcuquí, Ecuador; and Escuela de Medicina, Universidad Espíritu Santo, Samborondón, Ecuador

Mary Pulgar-Sánchez, Escuela Ciencias Biológicas e Ingeniería, Universidad Yachay Tech, Urcuquí, Ecuador

Kevin Chamorro, Escuela de Matemáticas y Ciencias

Computacionales, Universidad Yachay Tech, Urcuquí, Ecuador

Esteban Fernández-Moreira and Hégira Ramírez, Escuela de Medicina, Universidad de las Américas-UDLA, Quito, Ecuador Francisco X. Mora, IESS Hospital Quito Sur, Quito, Ecuador
}

largely relies on the clinical symptoms and computed tomography exams. In the hope to help risk-stratification and guide the timing of admission, some studies have also reported laboratory fluctuations in routine blood tests, which could become the mainstay for the forecasting of COVID-19 patients and the lessening of mortality [1]. Nevertheless, the characterization of the hematological and biochemical findings predicting COVID-19 severity are preliminary due to the low sample sizes, different proportions of severe patients, and geographic selection bias, and should therefore be taken with caution. Hematological biomarkers of COVID-19 severity requires validation by using larger samples of patients from different geographic localizations and ethnic groups across the globe.

We retrospectively analyzed the laboratory tests of 4,009 confirmed COVID-19 patients at the time of admission in the IESS Hospital Quito Sur in Quito (Ecuador) from March 13 to June 17, 2020. The hospital is the main COVID-19 medical center in the Quito metropolitan area, and it was ideally chosen given its privileged location at 2,850 m height over sea level. Considering the poor tolerance of the Andeans to high altitude illnesses [2], we described the hematological findings associated with COVID-19 severity in a population permanently exposed to the hypoxic environment induced by high altitude $(>2,500 \mathrm{~m})$. Written informed consent was waived due to the retrospective nature of the study. We followed the STROBE guidelines to conduct this study. In addition, we also had no access to identifying patient information.

The epidemiological analysis focused on 24 relevant laboratory variables including a hemogram, arterial blood gases, and some combined laboratory tests as biomarkers of systemic inflammation (Table 1). Hematological analysis were performed using a Sysmex XN-550 ${ }^{\mathrm{TM}}$ Hematology Analyzer (Sysmex America Inc., USA). Arterial blood gasometry was conducted on a RAPIDPoint ${ }^{\circledR} 500$ blood gas system (Siemens Healthcare GmbH; Germany). Normal 
Table 1: Hemogram and arterial blood gases on admission of COVID-19 patients.

\begin{tabular}{|c|c|c|c|c|c|c|c|}
\hline \multirow[t]{2}{*}{ Variable, units } & \multicolumn{2}{|c|}{ Non-severe } & \multicolumn{2}{|c|}{ Severe } & \multirow[b]{2}{*}{ p-Value } & \multirow[b]{2}{*}{ RBC } & \multirow[b]{2}{*}{ Reference values } \\
\hline & $\mathbf{n}$ & Mean $( \pm$ SD) & $\mathbf{n}$ & Mean $( \pm$ SD) & & & \\
\hline Hematocrit, \% & 3,215 & $43.4(5.4)$ & 459 & $43.9(5.6)$ & 0.046 & 0.058 & $38.4-47.3$ \\
\hline $\mathrm{RBC}, \cdot 10^{12} / \mathrm{L}$ & 3,192 & $5.1(0.6)$ & 454 & $5.2(0.6)$ & 0.002 & 0.092 & $4.5-5.7$ \\
\hline MCV, fL & 3,240 & $86.0(6.6)$ & 466 & $85.2(6.6)$ & 0.012 & 0.092 & $78-100$ \\
\hline RDW-SD, fL & 2,891 & $42.8(2.9)$ & 408 & $43.4(3.0)$ & $<0.001$ & -0.085 & $40-55$ \\
\hline Hemoglobin, g/dL & 3,225 & $14.9(2.0)$ & 463 & $15.4(2.0)$ & $<0.001$ & 0.148 & $13.0-16.1$ \\
\hline $\mathrm{MCH}, \mathrm{pg}$ & 3,125 & $29.7(1.8)$ & 457 & $29.7(1.8)$ & 0.521 & 0.124 & $26-34$ \\
\hline $\mathrm{MCHC}, \mathrm{g} / \mathrm{dL}$ & 3,269 & $34.4(1.9)$ & 471 & $34.8(2.0)$ & $<0.001$ & -0.014 & $32-36$ \\
\hline Platelets, $\cdot 10^{9} / \mathrm{L}$ & 3,192 & $258.9(73.6)$ & 459 & $234.7(74.7)$ & $<0.001$ & -0.384 & $150-400$ \\
\hline MPV, fL & 3,248 & $8.1(0.9)$ & 472 & $8.0(0.9)$ & 0.573 & 0.235 & $7.5-10.0$ \\
\hline WBC, $\cdot 10^{9} / \mathrm{L}$ & 3,152 & $7.7(2.9)$ & 441 & $7.9(3.1)$ & 0.216 & -0.011 & $4.5-11.0$ \\
\hline Neutrophils, $\cdot 10^{9} / \mathrm{L}$ & 3,143 & $5.0(2.7)$ & 430 & $5.8(2.8)$ & $<0.001$ & 0.025 & $2.5-8.0$ \\
\hline Lymphocytes, $\cdot 10^{9} / \mathrm{L}$ & 3,298 & $1.9(1.2)$ & 473 & $1.4(0.8)$ & $<0.001$ & -0.038 & $1-4$ \\
\hline Neutrophils, \% & 3,282 & $64.5(15.0)$ & 476 & $74.3(12.8)$ & $<0.001$ & 0.286 & $40-70$ \\
\hline Basophils, \% & 3,200 & $0.6(0.3)$ & 464 & $0.5(0.3)$ & $<0.001$ & 0.378 & $0-1$ \\
\hline Eosinophils, \% & 2,636 & $1.3(1.2)$ & 300 & $0.7(0.9)$ & $<0.001$ & -0.043 & $0-5$ \\
\hline Monocytes, \% & 3,212 & $7.0(2.4)$ & 466 & $6.8(2.9)$ & 0.129 & -0.164 & $0-7$ \\
\hline Lymphocytes, \% & 3,273 & $25.9(12.7)$ & 476 & $17.5(10.4)$ & $<0.001$ & -0.337 & $22-44$ \\
\hline NLR & 3,298 & $3.6(4.5)$ & 473 & $5.8(8.6)$ & $<0.001$ & -0.39 & $0.83-3.92$ \\
\hline PLR & 3,298 & $171.4(147.7)$ & 473 & $240.7(258.0)$ & $<0.001$ & -0.206 & 61-239 \\
\hline LeuCR & 1,755 & $4.4(11.0)$ & 352 & $0.96(3.5)$ & $<0.001$ & -0.386 & - \\
\hline LyCR & 1,714 & $1.6(4.7)$ & 346 & $0.5(2.4)$ & $<0.001$ & -0.436 & - \\
\hline $\mathrm{O}_{2}$ Sat, \% & 765 & $94.7(2.1)$ & 379 & $88.2(3.3)$ & $<0.001$ & -0.95 & $90-100$ \\
\hline $\mathrm{PaO}_{2}, \mathrm{mmHg}$ & 726 & $72.1(9.5)$ & 489 & $49.4(8.8)$ & $<0.001$ & -1.000 & $75-100$ \\
\hline $\mathrm{PaCO}_{2}, \mathrm{mmHg}$ & 751 & $30.5(4.9)$ & 462 & $32.9(5.2)$ & $<0.001$ & 0.244 & $38-42$ \\
\hline
\end{tabular}

Reference ranges: https://labtestsonline.org/articles/laboratory-test-reference-ranges. Alpha-value set at 0.05. Rank-Biserial Correlation $\left(r_{b c}\right)$ coefficient: $<0.10=$ trivial; $0.10-0.20=$ small; $0.21-0.40=$ medium; $\geq 0.50=$ large. RBC, red blood cell count; $M C V$, medium corpuscular volume; RDW-SD, red cell distribution width based on standard deviation; $M C H$, medium hemoglobin concentration; MCHC, medium corpuscular hemoglobin concentration; MPV, medium platelet volumen; WBC, white blood cell count, NLR, neutrophil-to-lymphocyte ratio; PLR, platelet-tolymphocyte ratio; LeuCR, leukocyte-to-C-reactive protein ratio; LyCR, lymphocyte-to-C-reactive protein ratio; $\mathrm{O}_{2}$ Sat, hemoglobin oxygen saturation; $\mathrm{PaO}_{2}$, partial arterial $\mathrm{O}_{2}$ pressure; $\mathrm{PaCO}_{2}$, partial arterial $\mathrm{CO}_{2}$ pressure.

distribution of the data was determined by the Kolmogorov-Smirnov test. Confirmed COVID-19 patients were categorized as severe (489 cases: $\mathrm{PaO}_{2}<60 \mathrm{mmHg} ; \mathrm{O}_{2}$ Sat $<90 \%)$ and non-severe (3,520 cases: 726 patients with $\mathrm{PaO}_{2} \geq 60 \mathrm{mmHg} ; \mathrm{O}_{2}$ Sat $\geq 90 \%$ in addition to 2,794 patients with a pneumonia score index below three) upon admission. The Mann-Whitney U test was conducted for statistical comparisons of non-parametric continuous variables between both Severe and Non-Severe groups, whereas the effect size was estimated by the Rank-Biserial Correlation coefficient $\left(r_{b c}\right)$. The demographic analyses of the dataset using the Chi-square test analysis revealed that the prevalence of severe COVID-19 pneumonia in males was greater than in females $\left(\chi^{2}(1)=28,382, p<0.001\right)$ and increased with age $\left(W=1.176 \cdot 10^{6}, \mathrm{p}<0.001\right)$, which was in agreement with prior studies [3]. As a disease reference, we also presented the laboratory tests of a group of 83 community acquired pneumonia (CAP) patients, which were performed on admission in the same hospital between November and December of 2018. It was split into Survivors and Non-Survivors groups based on patient mortality (Table 2). These laboratory results were only considered like a reference of the hematological findings found in regular pneumonia at high altitude.

Empowered by one of the largest sample size so far reported, this retrospective epidemiological study describes some peculiar characteristics of COVID-19 patients recruited at high altitude. Firstly, changes in the erythrogram parameters (Table 1) were unexpectedly trivial, at least at high altitude, and therefore not consistently associated with COVID-19 pneumonia severity (Table 1). A reduction of either hemoglobin or red blood cells (RBCs) were not observed in severe COVID-19 as reported by others $[1,3]$. Anemia is a determinant risk factor for not only COVID-19 severity [4, 5], but also for CAP in patients permanently living at high altitude 
Table 2: Hemogram and arterial blood gases on admission of CAP patients.

\begin{tabular}{|c|c|c|c|c|c|c|}
\hline \multirow[t]{2}{*}{ Variable, units } & \multicolumn{2}{|c|}{ Survivors } & \multicolumn{2}{|c|}{ Non-survivors } & \multirow[b]{2}{*}{ p-Value } & \multirow[b]{2}{*}{ Reference values } \\
\hline & n & Mean $( \pm$ SD) & $\mathbf{n}$ & Mean $( \pm$ SD) & & \\
\hline Hematocrit, \% & 65 & $42.0(8.8)$ & 18 & 36.7 (7.9) & 0.026 & $38.4-47.3$ \\
\hline $\mathrm{RBC}, \cdot 10^{12} / \mathrm{L}$ & 56 & $4.6(1.1)$ & 16 & $4(0.9)$ & 0.020 & $4.5-5.7$ \\
\hline $\mathrm{MCV}, \mathrm{fL}$ & 54 & $90.7(5.9)$ & 17 & $91(4.5)$ & 0.843 & $78-100$ \\
\hline RDW-SD, fL & 47 & $46.7(6.1)$ & 14 & $49.7(11.1)$ & 0.150 & $40-55$ \\
\hline Hemoglobin, g/dL & 63 & $14.0(2.9)$ & 17 & $12.2(2.6)$ & 0.021 & $13.0-16.1$ \\
\hline $\mathrm{MCH}, \mathrm{pg}$ & 54 & $30.3(2.5)$ & 15 & $30.4(1.5)$ & 0.856 & $26-34$ \\
\hline $\mathrm{MCHC}, \mathrm{g} / \mathrm{dL}$ & 54 & $33.3(1.2)$ & 15 & $33.2(1.1)$ & 0.305 & $32-36$ \\
\hline Platelets, $\cdot 10^{9} / \mathrm{L}$ & 63 & $222(105)$ & 18 & $248(118)$ & 0.315 & $150-400$ \\
\hline MPV, $\mathrm{fL}$ & 52 & $9.7(1.1)$ & 15 & $9.5(1.5)$ & 0.535 & $7.5-10.0$ \\
\hline WBC, $\cdot 10^{9} / \mathrm{L}$ & 65 & $9.2(3.9)$ & 18 & $9.2(5.1)$ & 0.749 & $4.5-11.0$ \\
\hline Neutrophils, $\cdot 10^{9} / \mathrm{L}$ & 54 & $7.7(4.0)$ & 16 & $8.1(4.8)$ & 0.983 & $2.5-8.0$ \\
\hline Lymphocytes, $\cdot 10^{9} / \mathrm{L}$ & 53 & $1.2(0.7)$ & 15 & $1.1(0.7)$ & 0.314 & $1.0-4.0$ \\
\hline Neutrophils, \% & 60 & $76.2(12.3)$ & 18 & $81.9(10.3$ & 0.087 & $40-70$ \\
\hline Basophils, \% & 53 & $0.3(0.3)$ & 16 & $0.4(0.4)$ & 0.474 & $0-1$ \\
\hline Eosinophils, \% & 56 & $1.1(1.6)$ & 16 & $0.9(1.2)$ & 0.973 & $0-5$ \\
\hline Monocytes,\% & 55 & $6.5(2.2)$ & 16 & $5.5(3.5)$ & 0.039 & $0-7$ \\
\hline Lymphocytes, \% & 58 & $15.5(10.6)$ & 17 & $11.9(6.6)$ & 0.366 & $22-44$ \\
\hline NLR & 53 & $9.1(7.8)$ & 15 & $11.1(8.7)$ & 0.482 & $0.83-3.92$ \\
\hline PLR & 54 & $216.2(145.6)$ & 15 & 337.4 (202) & 0.021 & 61-239 \\
\hline $\mathrm{O}_{2}$ Sat, $\%$ & 39 & $86.7(9.8)$ & 8 & $84.8(22.8)$ & 0.697 & $90-100$ \\
\hline $\mathrm{PaO}_{2}, \mathrm{mmHg}$ & 39 & $63.3(20.0)$ & 8 & $75.4(33.4)$ & 0.153 & $75-100$ \\
\hline $\mathrm{PaCO}_{2}, \mathrm{mmHg}$ & 39 & $33.4(7.5)$ & 8 & $33.6(10.9)$ & 0.989 & $38-42$ \\
\hline
\end{tabular}

Reference ranges: https://labtestsonline.org/articles/laboratory-test-reference-ranges. Alpha-value set at 0.05. RBC, red blood cell count; MCV, medium corpuscular volume; RDW-SD, red cell distribution width based on standard deviation; MCH, medium hemoglobin concentration; MCHC, medium corpuscular hemoglobin concentration; MPV, medium platelet volumen; WBC, white blood cell count, NLR, neutrophil-tolymphocyte ratio; $\mathrm{PLR}$, platelet-to-lymphocyte ratio; $\mathrm{O}_{2}$ Sat, hemoglobin oxygen saturation; $\mathrm{PaO}_{2}$, partial arterial $\mathrm{O}_{2}$ pressure; $\mathrm{PaCO}$, partial arterial $\mathrm{CO}_{2}$ pressure.

(Table 2). It was then surprising that the average hemoglobin levels in the Severe group was slightly higher compared to the Non-Severe group (Table 1), perhaps because of alteration of the hemoglobin mass [2]. The increase of the red width distribution (RDW) with COVID-19 severity [5] was trivial in our analysis. Some hypothesis should be put forward. Perhaps the high altitude-induced alteration of RDW [6] could specifically dampen RBCs and hemoglobin changes associated with COVID-19 severity, since it did not prevent anemia leading to fatal CAP outcomes (Table 2). A comparative study between the hematological findings in COVID-19 patients living at sea level with those residing at high altitude may shed some light on this hypothesis.

A second striking finding is that we found no significant differences in the counts of WBCs across Non-Severe and Severe groups as a metanalysis stated [1]. The reason is unknown, but an excessive neutrophil percentage in severe cases was likely to provoke a compensatory reduction in lymphocytes (Table 1) during the severe progression of COVID-19 [7]. In support of this hypothesis the neutrophil- to-lymphocyte ratio (NLR), a biomarker of systemic inflammatory response (Table 1) actually predicted admission to the intensive care unit in the patients of the Severe group [8]. Of note, COVID-19 severity was not associated with a significant reduction of the percentage of monocytes as it was the case of CAP (Table 2). Another inflammation biomarker, the platelet-to-lymphocyte ratio (PLR) predicted illness severity either in COVID-19 or in CAP patients living at high altitude. Finally, the leukocyte-to-C-protein ratio (LeuCR), a novel composed laboratory test parameter based on prior evidence [7], showed the largest reduction with COVID-19 severity, so that its potential as a biomarker of systemic inflammation in COVID-19 awaits future research.

In summary, even if there is no doubt that specific hematologic fluctuations contribute to the worst clinical outcomes of COVID-19 [1], largely those denoting a hyperinflammation state $[9,10]$, the influence of the erythrogram changes were unexpectedly trivial in patients suffering from severe COVID-19 at high altitude. This posits the intriguing hypothesis that hematological fluctuations associated with the exposure to high altitude-induced 
hypoxia could have an impact in the progression of coronavirus disease to ARDS.

Research funding: None declared.

Author contributions: All authors have accepted responsibility for the entire content of this manuscript and approved its submission.

Competing interests: Authors state no conflict of interest. Ethical approval: Not applicable.

\section{References}

1. Alnor A, Sandberg MB, Gils C, Vinholt PJ. Laboratory tests and outcome for patients with Coronavirus disease 2019: a systematic review and meta-analysis. J Appl Lab Med 2020;5:1038-49.

2. Sánchez K, Ballaz SJ. Might a high hemoglobin mass be involved in non-cardiogenic pulmonary edema? The case of the chronic maladaptation to high-altitude in the Andes. Med Hypotheses 2021;146:110418.

3. Yuan X, Huang W, Ye B, Chen C, Huang R, Wu F, et al. Changes of hematological and immunological parameters in COVID-19 patients. Int J Hematol 2020;112:553-9.
4. Tao Z, Xu J, Chen W, Yang Z, Xu X, Liu L, et al. Anemia is associated with severe illness in COVID-19: a retrospective cohort study. J Med Virol 2020;93:1478-88.

5. Lippi G, Henry BM, Sanchis-Gomar F. Red blood cell distribution is a significant predictor of severe illness in Coronavirus disease 2019. Acta Haematol 2020:1-5. https://doi.org/10.1159/ 000510914 [Epub ahead of print].

6. Han K, Su X, Liu J, Yao F, Lu F. Red cell distribution width as a novel marker for different types of atrial fibrillation in low and high altitude. Cardiol Res Pract 2019;2019:6291964.

7. Yamada T, Wakabayashi M, Yamaji T, Chopra N, Mikami T, Miyashita $\mathrm{H}$, et al. Value of leukocytosis and elevated C-reactive protein in predicting severe coronavirus 2019 (COVID-19): a systematic review and meta-analysis. Clin Chim Acta 2020;509: 235-43.

8. Ciccullo A, Borghetti A, Zileri Dal Verme L, Tosoni A, Lombardi F, Garcovich M, et al. Neutrophil-to-lymphocyte ratio and clinical outcome in COVID-19: a report from the Italian front line. Int J Antimicrob Agents 2020;56:106017.

9. Wang J, Li Q, Yin Y, Zhang Y, Cao Y, Lin X, et al. Excessive neutrophils and neutrophil extracellular traps in COVID-19. Front Immunol 2020;11:2063.

10. Zheng M, Gao Y, Wang G, Song G, Liu S, Sun D, et al. Functional exhaustion of antiviral lymphocytes in COVID-19 patients. Cell Mol Immunol 2020;17:533-5. 